\title{
Symposium
}

\section{Symposium on Treatment of Cancer*}

\author{
Moderator: Kyoshi Konno, MD \\ Department of Internal Medicine, The Research Institute for Chest Diseass \\ and Cancer, Tohoku University, Sendai \\ Moderator: Kunio OKUdA, MD \\ The First Department of Internal Medicine, School of Medicine, \\ Chiba University, Chiba

\section{Chemotherapy of Acute Leukemia in Adults} \\ Kazumasa YAMADA, MD \\ Department of Medicine, The Branch Hospital, \\ Nagoya University School of Medicine
}

Clinical effects of new agents in the treatment of leukemia and lymphoma are presented.

1) $\mathrm{N}^{4}$-Behenoyl-1- $\beta$-D-arabinofuranosylcytosine

$(\mathrm{BH}-\mathrm{AC})^{1)}$

In phase II study of BH-AC, significant responses were noted in AML patients with a CR rate of $44.1 \%$.

A phase III study of BH-AC in adult with ANLL was conducted by a Cooperative Study Group of the Treatment of Leukemia.

The regimen used and dosage schedules of

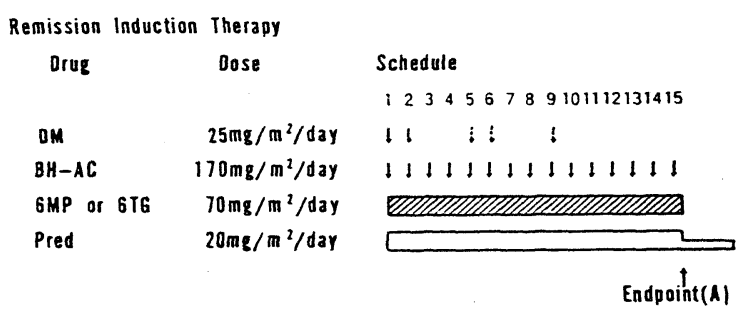

Fig. 1. BH-AC·DMP Protocol for Adult ANLL each drug were as follows: $\mathrm{BH}-\mathrm{AC}, 170 \mathrm{mg} / \mathrm{m}^{2}$ per day, daunorubicin (DM) $25 \mathrm{mg} / \mathrm{m}^{2}$ per day, 6-mercaptopurine (6-MP), $80 \mathrm{mg} / \mathrm{m}^{2}$ per day, and prednisolone (pred), $20 \mathrm{mg} / \mathrm{m}^{2}$ per day, both given orally (BH-AC·DMP regimen $)^{2}$. (Fig. 1)

The response of the patients to the BH-AC. DMP regimen is shown in Table 1. Sixty-five $(74.7 \%)$ of the 87 patients with acute myeloblastic leukemia (AML), $29(69.0 \%)$ of the pa-

Table 1. Effects of BH-AC.DMP Therapy in Adult ANLL

\begin{tabular}{lrrrrr}
\hline Type & $\begin{array}{l}\text { No. of } \\
\text { Cases }\end{array}$ & CR & PR & F & $\begin{array}{l}\text { CR } \\
\text { Rate(\%) }\end{array}$ \\
\hline AML & 87 & 65 & 2 & 20 & 74.7 \\
AProL & 12 & 9 & 0 & 3 & 75.0 \\
AMoL & 42 & 29 & 6 & 7 & 69.0 \\
\hline Total & 141 & 103 & 8 & 30 & 73.1 \\
\hline
\end{tabular}

*Presented at the 81st Annual Meeting of the Japanese Society of Internal Medicine, April 3, 1984, in Fukuoka. 
tients with acute myelomonocytic leukemia (AMOL), and $9(75.0 \%)$ of the 12 patients with acute promyelocytic leukemia (AproL) achieved complete remission (CR), respectively. Overall, $103(73.1 \%)$ of the 141 patients with ANLL responded with complete remission. (Table 1)

The results obtained with the BH-AC-DMP therapy are superior to those in previous reports ${ }^{3)}$, in terms of rate of complete remission, remission duration and survival.

2) $\mathrm{N}^{4}$-palmitoyl-arabinofuranosylcytosine

\section{$(\text { PLAC) })^{4)}$}

Forty-six adults with acute leukemia were entered in phase II study of oral use of PLAC. PLAC $100-200 \mathrm{mg} / \mathrm{m}^{2} / \mathrm{d}$ was given orally for more than 30 days.

Responders with CR were noted with an overall rate of $10.1 \%$, and those with PR were $15.9 \%$. Of the patients who received PLAC orally, 5 of 30 patients $(12.5 \%)$ with AML and 1 of 6 with BC of CML (16.6\%) responded with CR. Two of 30 with AML, 3 of 6 with AMoL and each one of 3 with ALL and of 6 with CML-BC responded with PR.

Oral use of PLAC is therapeutic in the treatment of AML, particularly for atypical type of the disease.

3) Aclacinomycin A (ACM-A)

After phase I trial in human, phase II-III study of ACM-A in adult acute leukemia was conducted by a Cooperative Study Group. In phase II study, $38 \%$ of 21 patients responded with $\mathrm{CR}$.

A phase III study of ACM-A in combination with BH-AC, 6-MP and prednisolone has been initiated (BH-AC·AMP regimen). The dosage schedule of each drug was as follows: ACM-A, $14 \mathrm{mg} / \mathrm{m}^{2}$ per day intravenous infusion, BH-AC, $170 \mathrm{mg} / \mathrm{m}^{2}$ per day intravenously, 6-MP, 80 $\mathrm{mg} / \mathrm{m}^{2}$ per day orally, and prednisolone, $20 \mathrm{mg} /$ $\mathrm{m}^{2}$ per day orally.

Of the 60 previously untreated patients, $66.7 \%$ responded with $\mathrm{CR}$. There are $27 \mathrm{CRs}$ in 37 patients with AML, $10 \mathrm{CRs}$ in 14 patients with AMoL, 2 CRs in 3 patients with acute promyelocytic leukemia (APL) and $1 \mathrm{CR}$ in 6 patients with erythroleukemia (EL).

Of the 65 patients who had had prior treatment with chemotherapy, $66.7 \%$ responded with CR. There were $25 \mathrm{CRs}$ in 41 patients with AML, $5 \mathrm{CRs}$ in 10 patients with AMoL, and 11 CRs in 13 patients with APL. BH-AC·AMP therapy produced encouraging results in terms of response rate, particularly in patients who had hd prior chemotherapy, since most of the responders were considered to be resistant to multicombination chemotherapy (BH-AC·DMP regimen $)^{6)}$.

4) Tetrahydropyranil-adriamycin (THP-ADM)

THP-ADM is less toxic, particularly less cardiotoxic than other anthracyclines currently being clinically used and it has superior antitumor activity in experimental tumor systems to ADM.

The dose schedules employed were as follows: $15-20 \mathrm{mg} / \mathrm{m}^{2}$ daily i.v. for 3 to 5 days for patients with acute leukemia, and 40 to $50 \mathrm{mg} / \mathrm{m}^{2}$ once every 3 weeks i.v. for those with malignant lymphoma.

Each one of 6 patients with ALL and of 12 patients with AML achieved CR. Five of 22 patients had PR. Out of 19 evaluable patients with non-Hodgkin's lymphoma, 3 had $\mathrm{CR}$ and 5 had PR, response rate being $42.1 \%$.

THP deserves further prospective study in leukemias and lymphomas.

5) Human Lymphoblastoid Interferon (HLBI)

The phase II clinical trial of HLBI in patients with multiple myeloma, leukemias and lymphomas was conducted by a Cooperative Study Group in Japan involving 24 institutions during a two year period.

Most of the patient received 3 mega international units of HLBI I.M. daily and in some patients, the dose was escalated to 6-12 mega units over a period of at least 4 weeks.

Of 73 evaluable patients with multiple myeloma, $1 \mathrm{CR}$ and $13 \mathrm{PR}$ cases were obtained which makes $19.2 \%$ of response rate. Of 53 patients with lymphomas including adult $\mathrm{T}$ cell leukemia, 2 CRs and 7 PRs occurred which makes $17.3 \%$ of remission rate. There were no $\mathrm{CR}$ in patients with leukemias, yet, one of 33 patients with acute leukemia and 2 of 9 patients with chronic leukemia responded with PR.

Of 32 patients with non-Hodgkin's lymphoma, $1 \mathrm{CR}$ and $2 \mathrm{PR}$ were seen, and it is noteworthy that of 10 patients with adult $\mathrm{T}$ cell leukemia, 
1 responded with $\mathrm{CR}$ and 2 responded with PR. HLBI deserves undoubtedly further prospective phase II-III clinical trials in multiple myeloma and lymphomas.

\section{REFERENCES}

1) Yamada $\mathrm{K}$, et al: Pharmacologic and clinical studies of $\mathrm{N}^{4}$-Behenoyl-1- $\beta$-D-arabinofuranosylcytosine. Recent Results in Cancer Research 70: 219-229, 1980.

2) Yamada K, et al: Phase III study of $\mathrm{N}^{4}$-behenoyl$1-\beta$-D-arabinofuranosylcytosine in adult acute leukemia. Current Chemotherapy and Immunotherapy
II: $1308-1310,1982$.

3) Yamada K, et al: DCMP two-step therapy for acute myelogenous leukemia in adults. Jpn J Clin Oncol 8: 133-140, 1978.

4) Tsuruo T: Comparison of cytotoxic effect and cellular uptake of 1- $\beta$-D-arabinofuranosylcytosine and its $\mathrm{N}^{4}$-acyl derivatives using cultured $\mathrm{KB}$ cells. Cancer Research 39: 1063-1070, 1979.

5) Yamada K, et al: A phase II study of aclacinomycin $A$ in acute leukemia in adults. Cancer Treatment Review 7: 177-182, 1980.

6) Umezawa $\mathrm{H}$, et al: Tetrahydropyranil derivatives of daunomycin and adriamycin. J Antibiotics 32: 1082-1084, 1979 .

\title{
2. Alternating Combination Chemotherapy with a Multimodality Approach for Small Cell Lung Cancer
}

\author{
Ikuro KImura, MD and Taisuke OHNOSHI, MD
}

The Second Department of Medicine, Okayama University Medical School

Small cell lung cancer (SCLC) is disseminated at the presentation in most patients. Chemotherapy is, therefore, the principal therapeutic modality in this disease. The application of aggressive chemotherapies has resulted in improved survival for patients with SCLC. Our previous study utilizing a four-drug combination of cyclophosphamide, vincristine, methotrexate, and procarbazine $(\mathrm{COMP})^{1)}$ resulted in; 1$)$ response rate of $68 \%$ with a complete remission (CR) rate of $28 \%, 2)$ an overall median survival time of 9.5 months, with a significant prolongation of life in patients who achieved $C R, 3$ ) a fraction of long-term disease-free survivors, especially in those who had received concomitant chest irradiation, and 4) a high frequency (41\%) of cerebral metastases in complete responders.

The current study outlined in Fig. 1 was undertaken to test a cyclic alternating chemotherapy with the four-drug combination of COMP (cyclophosphamide, $270 \mathrm{mg} / \mathrm{m}^{2}$, on day 1-5; vincristine, $1.4 \mathrm{mg} / \mathrm{m}^{2}$, on day 1 ; methotrexate, $6.5 \mathrm{mg} / \mathrm{m}^{2}$, on day $1-5$; and procarbazine, 65 $\mathrm{mg} / \mathrm{m}^{2}$, on day $1-5$ ) and a three-drug combina- tion of VAN (oral VP-16, $140 \mathrm{mg} / \mathrm{m}^{2}$, on day 29-32; adriamycin, $40 \mathrm{mg} / \mathrm{m}^{2}$, on day 29; and ACNU $40 \mathrm{mg} / \mathrm{m}^{2}$, on day 29) which appeared non-cross-resistant to COMP. In this study, also included two randomized clinical trials to test the effectiveness of concomitant chest irradiation in patients with limited disease (LD) who received the intensive alternating chemotherapy, and to test the validity of prophylactic cranial irradiation (PCI) for patients who achieved CR.

Between March, 1981 and November, 1983, 69 patients with SCLC entered the study. After stratification for the extent of disease, $34 \mathrm{pa}$ tients with $L D$ were randomized to receive the alternating chemotherapy alone or to receive chest irradiation after the first cycle of COMP and VAN. Thirty-five patients with extensive disease (ED) received the chemotherapy alone. Twenty-eight patients who had achieved CR were randomized to receive PCI or to have observation only. Of 35 patients with ED, 13 (37\%) achieved CR and 18 (51\%) achieved partial remission (PR). The projected median survival time was 8.5 months for all patients with ED. Of 18 pa- 